A Castañeda Zumeta y P Pineda-Martínez (2016): "La reivindicación democrática a través de las viñetas. Las temáticas del humor gráfico en el periódico vasco Egin (1977)". Revista Latina de Comunicación Social, 71, pp. 232 a 248.

\title{
La reivindicación democrática a través de las viñetas. Las temáticas del humor gráfico en el periódico vasco Egin (1977)
}

\section{The promotion of democracy in political cartoons. The themes of cartoons in the Basque newspaper Egin (1977)}

\begin{abstract}
Aitor Castañeda Zumeta [ $\underline{\mathrm{CV}}$ ] [ORCID] [ $\mathrm{C}$ GS $]$ Departamento de Comunicación Audiovisual y Publicidad, Universidad del País Vasco del País Vasco (UPV/EHU) - aitor.castaneda@ehu.eus

Paula Pineda-Martínez [CV] [ORCID] [ $\mathrm{C}$ GS] Departamento de Comunicación Audiovisual y Publicidad, Universidad del País Vasco del País Vasco (UPV/EHU) - paula.pineda@ehu.eus
\end{abstract}

\begin{abstract}
s
[ES] Introducción. El estudio pretende identificar las temáticas empleadas en las tiras cómicas del periódico vasco Egin (1977/98) durante el primer año de su existencia. El diario, de tendencia "abertzale", resultó de gran impacto en la sociedad vasca de la Transición por ser el primero en encuadrarse plenamente en aquel espectro social de la izquierda, y por dedicar toda una página al humor gráfico. Metodología. Se lleva a cabo un análisis temático de las tiras cómicas que se encuentran en la página de entretenimiento del diario durante todo 1977. Del mismo modo, se emplean técnicas cuantitativas para medir la frecuencia de las temáticas identificadas. Resultados y conclusiones. Los resultados muestran una clara preferencia de los autores por los temas relacionados con la reivindicación democrática, así como la muestra de un contrapunto entre las tesis del nacionalismo vasco tradicional contra un nuevo nacionalismo que se está gestando a través de la izquierda.

[EN] Introduction. This article aims to identify the different themes explored in the comic strips published by the Basque newspaper Egin (1977/98) during its first year of operations. This nationalist newspaper had a great impact on the Basque society during the Spanish transition to democracy because it was the first left-wing publication in the Basque Country and because it devoted a whole page to graphic humour. Method. The study is based on the thematic analysis of the comic strips published in the entertainment section of the aforementioned newspaper throughout 1977. Quantitative techniques were used to identify and measure the frequency of occurrence of the diversity of issues and themes. Results and conclusions. The results show the clear preference of
\end{abstract}


cartoonists for issues related to the promotion of democracy, as well as the existence of an opposition within the premises of traditional Basque nationalism against the new form of nationalism promoted by leftist movements.

\section{Keywords}

[ES] Democratización; humor gráfico; prensa; identidad local; mirada crítica; producción artística. [EN] Democratisation; graphic humour; press; local identity; critical view; artistic production.

\section{Contents}

[ES] 1. Introducción. 1.1. El objeto de estudio. 1.2. Objetivos. 1.3. Interés del diario Egin y su humor gráfico. 1.3.1. Contexto sociopolítico del País Vasco y Navarra a finales de los 70. 1.3.2. La página "Solasjaipausa". 1.4. El humor gráfico como disciplina académica. 2. Material y estrategias metodológicas. 2.1. Algunas definiciones previas. 2.2. Metodología. 2.2.1. Muestra. 2.2.2. Apartado cualitativo. 2.2.3. Apartado cuantitativo. 3. Resultados. 4. Discusión y conclusiones. 5. Notas. 6. Referencias bibliográficas

[EN] 1. Introduction. 1.1. Object of study. 1.2. Objectives. 1.3. The interests and graphic humour of Egin. 1.3.1. Socio-political context of the Basque Country and Navarre in the late 1970s. 1.3.2. The "Solasjaipausa" section. 1.4. Graphic humour as academic discipline. 2. Materials and methodological strategies. 2.1. Previous definitions. 2.2. Methods. 2.2.1. Sample. 2.2.2. Qualitative methods. 2.2.3. Quantitative methods. 3. Results. 4. Discussion and conclusions. 5. Notes. 6. References.

Traducción de CA Martínez Arcos (Dr. en Comunicación por la Universidad de Londres)

\section{Introducción}

\subsection{El objeto de estudio}

El humor gráfico (en adelante HG), género periodístico nacido en la segunda mitad del siglo XIX, tiene una larga tradición en España. Autores como Iván Tubau lo definen como "el mejor del mundo" (Tubau, 1973). Y es que, desde la perspectiva de las comunicaciones sociales, se trata de un género periodístico de opinión (autores como Armañanzas y Díaz Noci lo denominan también "opinión iconográfica") por medio del cual pueden expresarse determinadas ideas que de otro modo resultarían difíciles de asumir.

"En una publicación, la opinión no sólo puede aportarse a través de textos, sino que también puede darse a conocer a través de ilustraciones (...). En las páginas de Opinión de un diario las ilustraciones son bien de carácter iconográfico - retratos, caricatura- o viñetas de humor, con o sin texto. Si un simple dibujo en apariencia inocente puede estar cargado de intención, ésta se torna opinión crítica irónica, ácida, cuando viene acompañada del humor. Son esas viñetas que generalmente reposan a los pies de los editoriales o haciendo compañía a otros artículos de opinión en unas páginas fundamentales para conocer el posicionamiento de un periódico sobre los más variados acontecimientos”. (Armañanzas \& Díaz Noci, 1996: 111)

Resultan en España harto conocidas aquellas ilustraciones que ya en el Madrid del siglo XIX fueron publicadas en almanaques de difusión clandestina por mano de los hermanos Bécquer, recopiladas en una publicación de título "Los Borbones, en pelota", que no vio la luz hasta 1991, y que mostraba caricaturas e ilustraciones pornográficas de la corte de Isabel II. Así también, el investigador Ramón 
Reig nos recuerda algunas curiosas anécdotas acaecidas en la España franquista como consecuencia del HG:

"Cuando ejercía de ministro del Interior Manuel Fraga Iribarne y de alcalde de Barcelona Joaquín Viola (...) los Reyes de España, don Juan Carlos y doña Sofía, visitaron la llamada ciudad condal. Una publicación humorística tituló más o menos de esta forma: "Fraga recibe al Rey y Viola a la Reina”. Sufrió un secuestro. Algún tiempo antes, el semanario La Codorniz, estando de ministro (...) Laureano López Rodó, publicó una tira humorística en portada. Un campesino, supuestamente llamado López, subía por una montaña o colina cargando con un cerdo (...). De pronto, sus fuerzas flaquean, el cerdo cae de sus hombros y se va montaña abajo. La tira titula al final de su última viñeta de esta manera. "Y el cerdo de López rodó”. Más problemas con la censura” (Reig, 2008: Introducción, párrafo 1)

Del mismo modo, en los tiempos modernos, somos testigos de las diversas andanzas de la revista $E l$ Jueves con(tra) la Corona Española, así como el sangriento atentado del año 2015 contra la revista satírica francesa Charlie Ebdo. Conflictos, todos ellos, provocados por la 'amenaza' de dibujos e ilustraciones.

Y es que el HG como fenómeno social no sólo pretende simplemente hacer reír, sino muy a menudo sublimar situaciones personales de quien lo practica, a fin de liberarse de una carga interna que de otro modo no superaría.

Uno de los más destacados autores en las teorías estéticas, el alemán Juan Pablo Richter, quien tiene además un ensayo referente sobre el humorismo, afirma que después de cada tensión patética, el hombre experimenta ordinariamente la necesidad del descanso que el humor proporciona (Richter, 1812: 147). Del mismo modo, el doctor español Gil Fernández, quien estudia la comedia aristofánica, concluye:

"En lo cómico se da una especie de reacción compensatoria a un complejo de inferioridad, mediante la autoafirmación de la realidad propia. Para subsistir en condiciones adversas, el esclavo necesita alguna vez burlarse de sus amos: el hombre, de los dioses; el mediocre, de la superioridad intelectual; el simple ciudadano, de los políticos que lo gobiernan. Y con la risa se descargan tensiones que, de otro modo, llegarían a hacerse insoportables" (Gil Fernández, 1996: 18)

Para terminar, el profesor gallego Siro López, en un libro que precede al ensayo de Celestino Fernández de la Vega $O$ segredo do humor ("El secreto del humor"), deduce:

"O humorismo más sutil provén das minorías oprimidas que se valeron del para aturar a súa difícil existencia (...). A xente que sofre ou que sufriu adoita posuír mecanismos de defensa que se manifestan non maior talento para usar o humorismo como evasión" (López, 2005: 22)

Si todo esto es así, no cabe duda de que la presión autoritaria practicada a través de los siglos puede haber modelado en España un pueblo de un acusado sentido del humor, también reflejado entre sus mejores dibujantes a través de su opinión iconográfica. En ello nos vamos a centrar, tomando como punto de partida uno de los territorios política y socialmente más conflictivos del estado: el vasco. Y lo vamos a hacer tomando la socialmente tensa época de la Transición en el año 77, y analizando el HG de uno de los diarios más combativos que han existido en la región: Egin. 


\subsection{Objetivos}

a) Realizar un primer acercamiento al HG del periódico Egin a través del año 1977, identificando los temas que los dibujantes tratan en sus viñetas.

b) Identificar aquellas temáticas más frecuentes en cada autor.

c) Comprobar si la ideología del periódico tiene relación con los temas empleados por los dibujantes.

\subsection{Interés del diario Egin y su humor gráfico}

Egin ("hacer" o "haciendo" en vascuence) fue un diario nacionalista de tendencia de izquierda, bilingüe, cuyo primer ejemplar fue publicado el 29 de septiembre de 1977 de mano de la editorial guipuzcoana Orain S.A. (Hernani, Guipúzcoa). Su aparición trastocó, junto con Deia ("llamada"), el panorama periodístico vasco, en primer lugar, por tratarse ambos de periódicos que cambiaban el ámbito geográfico de difusión; si hasta el momento no podía hablarse de "prensa vasca" como tal [01], entendida como un espacio geográfico que abarcase como mínimo las provincias vascongadas, Egin fue periódico nacido con ediciones en las Provincias y Navarra, como Deia, pero el primero contaba además con una delegación en el País Vascofrancés, cubriendo así el espacio culturalmente denominado Euskalerría [02].

“A los que piensan que Euskal Herria, nuestro País Vasco -Álava, Baja Navarra, Guipúzcoa, Laburdi, Navarra, Vizcaya y Zuberoa- no solamente tienen una historia común en tantos elementos esenciales, sino también un presente que aclarar y un futuro que hacer conjuntamente, para bien de todos" (Egin, portada del 29/09/1977)

Se trataba, pues, de periódicos nacionalistas con objeto de 'hacer país'. Del mismo modo, mientras Deia era un diario afín al Partido Nacionalista Vasco (PNV), Egin lo fue del amplio espectro de la izquierda abertzale (en adelante IA), que dedicaba amplios espacios a dar voz a determinados colectivos hasta entonces acallados por la prensa oficial.

“[Egin] intenta captar especialmente a un público joven, situado próximo a la órbita de la izquierda abertzale, y deja bien claro que dedicará grandes espacios a informar sobre asuntos tradicionalmente abandonados por otros medios: mujer, ecología, marginalidad, etc." (Coca \& Martínez, 1993: 69)

Su repercusión en el ámbito de difusión fue también contundente entre los periódicos de entonces, de los cuales sólo se adaptaron a los nuevos tiempos El Correo Español - El Pueblo Vasco y El Diario Vasco, actuales decanos en Vizcaya y Guipúzcoa, respectivamente. Egin llegó a tener, en 1980, una tirada 41.400 ejemplares diarios (OJD, 1981), si bien su techo fue siempre de unos 50.000 (Mainer \& Juliá, 2000: 214).

Respondía, al principio, al multifacético espectro de la IA, si bien el mismo año de su salida se produjo una división en su consejo editorial debido a un atentado que ETA perpetró en Pamplona [03]. La condena del mismo supuso la dimisión del entonces director Mariano Ferrer, y la posterior radicalización de periódico, convirtiéndose en portavoz de la después ilegalizada "Herri Batasuna" 
(Coca \& Martínez, 1993: 31). Fue clausurado por la Audiencia Nacional de España el 15 de julio de 1998, por considerarlo vinculado a la banda terrorista, si bien posteriormente la acusación fue desestimada, sin que el periódico volviese a renacer.

\subsubsection{Contexto sociopolítico del País Vasco y Navarra a finales de los 70}

Atendiendo a los datos aportados por Coca y Martínez, puede constatarse que los años 70 son especialmente conflictivos en el País Vasco:

"Los años setenta fueron particularmente tensos. En el convulsionado País Vasco más que en ninguna otra parte, donde, a las actuaciones de ETA, había que añadir el soterrado y fuerte movimiento antifranquista y las frecuentes movilizaciones populares, siempre prohibidas y por tanto reprimidas por la Policía, los periodistas sufrían más de cerca la impotencia de ver circular en los medios de comunicación las versiones controladas y amañadas en los Gobierno Civiles". (Coca \& Martínez, 1993: 18)

La represión del dégimen comienza a debilitarse durante los últimos años de la dictadura, lo que contrasta con el clamor social por derrocar al caudillo. En el País Vasco ya ha tomado cuerpo la banda terrorista ETA, quien asesina a Carrero Blanco en 1973. El avance hacia la democracia va a resultar especialmente escabroso, en tanto que no queda claro hacia dónde avanza el nuevo régimen, lo que se irá dilucidando poco a poco conforme se llegue a la Transición, periodo en el que se enmarca esta investigación.

El 15 de diciembre de 1976, Adolfo Suárez convoca elecciones generales para la llamada Reforma Política, que allanará el camino jurídico hacia una nueva constitución. El amplio apoyo social a esta reforma desembocará el 16 de enero del 77 en las primeras elecciones generales con diputados a Cortes, en las que el partido de Suárez resultará ganador.

Pero a pesar de los esfuerzos políticos por avanzar hacia la democracia, quedan algunas inercias de la reciente dictadura. A nivel social, en el País Vasco ya habían empezado a salir a la calle movimientos en pro de la amnistía para los presos políticos, a los que hay que añadir constantes manifestaciones obreras, de las que cabe destacar la del 3 de marzo del 76, en la que las fuerzas policiales del Estado se saldaron en Vitoria con 5 obreros muertos y más de cien heridos en una asamblea de trabajadores en huelga.

El grupo editorial 'Prensa del Movimiento' seleccionaba y filtraba las informaciones periodísticas, con ayuda de los gobiernos civiles, aún sin democratizar. Ellos seleccionan las versiones de los hechos y su difusión a través de la prensa oficial, siendo la agencia estatal Cifra la única que provee de información a los medios a nivel nacional. Concretamente, la Asociación de Periodistas de Vitoria hizo público su malestar por la versión de los hechos del 3 de marzo que difundió la prensa oficial (Díaz Noci, 2012: 199). Conforme la libertad de prensa va haciéndose palpable, florecen, como Egin y Deia, publicaciones contrarias al régimen, que también reciben atentados de grupos de extrema derecha, como es el caso de la revista Punto y Hora de Euskal Herria, editada en Pamplona y atacada en octubre del 77. A nivel nacional, no pueden dejarse sin mencionar los atentados contra la revista satírica El Papus en septiembre del mismo año, también por grupos de extrema derecha.

A este enmarañado contexto sociopolítico, hay que añadir el factor de la identidad nacional disputada en el País Vasco. Especial atención merece el caso navarro. Como se verá más adelante, el conflicto 
territorial será especialmente complicado de resolver en este territorio, en tanto que su Diputación Foral, en manos de los tradicionalistas, y los diputados a cortes elegidos en 1977 por UCD ( 3 de 5 en total) se oponen tajantemente a compartir un estatuto propio con las Vascongadas, en aras de mantener su régimen foral, en oposición a las fuerzas nacionalistas y de izquierdas, que pretenden la unión político-administrativa de ambas regiones. La problemática, que dura hoy en día, tendrá una especial fuerza en los momentos en que se dé el salto al actual estado de las autonomías, donde finalmente Navarra quedará fuera del Estatuto de Guernica (1979). Las referencias a esta cuestión son una constante en el caso de Egin, como posteriormente se verá.

1977 es, por tanto, un año en el que queda poco para dar el salto hacia la Constitución vigente, y en el que las inercias del anterior régimen siguen haciendo fuerza para, si no detener, ralentizar el proceso democratizador del país. En el País Vasco, el conflicto territorial, las tensiones obreras, la falta de libertad de expresión y una sociedad dividida en lo político van a hacer, de Egin, un periódico cuyos contenidos serán de especial interés académico durante el año seleccionado.

\subsubsection{La página Solasjaipausa}

Sin duda uno de los aspectos más interesantes de este periódico fue la página Solasjaipausa ("Divertimentos"), donde se dedicaba toda una página al HG. Cabe mencionar que muchos lectores comenzaban la lectura del periódico por el reverso, donde se encontraba la misma (Lorente, 2015: 532). No hay que olvidar que la democracia aún no estaba consolidada, de tal manera que revistas de HG como el mencionado El Papus sufrieron cierres y atentados por parte de grupos de extrema derecha. La apuesta de Egin por este género es, pues, altamente arriesgada.

Un inmediato antecedente de "Solasjaipausa" la encontramos en la revista éuscara Anaitasuna ("Hermandad"), que incluía una página con viñetas y pasatiempos de título Zapaburua ("El Renacuajo"), en la que ya dibujaban los artistas que se suman después a Egin. Es el caso de los entonces muy conocidos caricaturistas Juan Carlos Eguillor (bilbaíno cuyas ilustraciones ya eran difundidas en el cartelismo y la prensa) y Jon Zabaleta, dibujante guipuzcoano que también había destacado como ilustrador de cuentos y material didáctico para el aprendizaje del euskera. Antonio Olariaga, quien había creado al personaje "Kaleko" ("Callejero") para Anaitasuna, comenzaría en Egin su recorrido como dibujante en un periódico de ámbito vasco, ya que hasta entonces había trabajado como dibujante e ilustrador en el diario Las Provincias, de Valencia (GFA, 1998, párrafo 1).

Fue Olariaga quien creó el popular personaje "Zakilixut" ("Pene-tieso"), que aún sigue publicando en la contraportada del diario vasco-parlante Berria, y diseñó la estructura de la propia página. Encabezaba la misma una tira cómica de Zabaleta, con su serie Barraskiloa eta Elefantea ("El elefante y el caracol"). En ella representa, con un estilo de línea curva y toque infantil, un mundo onírico donde elefantes y caracoles interactúan, normalmente de manera muda, dando a entender relatos con mensajes a veces no tan inocentes.

A Zabaleta le sigue Eguillor, con una serie castellana de nombre eusquérico "Beltza-Superbeltza" ("Negro-Supernegro"), donde satiriza la extensa tipología urbana de la villa de Bilbao a través de los movimientos de masas y sus alocados personajes, quienes se expresan en un español plagado de vasquismos. Tras él, Olariaga incluye su serie Zakilixut, donde el personaje principal, un joven solitario y obsesionado con el sexo, ironiza todo cuanto ve haciendo paralelismos con figuras obscenas. 
Junto a estos dibujantes, y al lado de la tira cómica de Eguillor, el periodista Ángel Lertxundi redacta, con ayuda de Olariaga, un texto humorístico diario en lengua vasca, de título "Juana Bixenta Olabe, parlamentarien neskame" ("Juana Vicenta Olabe, criada de los parlamentarios"). El personaje de Juana Bixenta Olabe (JBO) escribe a los parlamentarios que se agruparán en torno al Consejo General Vasco, encargado de redactar el primer estatuto de autonomía; con un particular tono sarcástico critica las actividades de sus dueños y la situación política del momento. Es un texto de carácter editorial, en tanto que Egin no hacía uso de este texto de opinión, logrando así una sensación de mayor aperturismo.
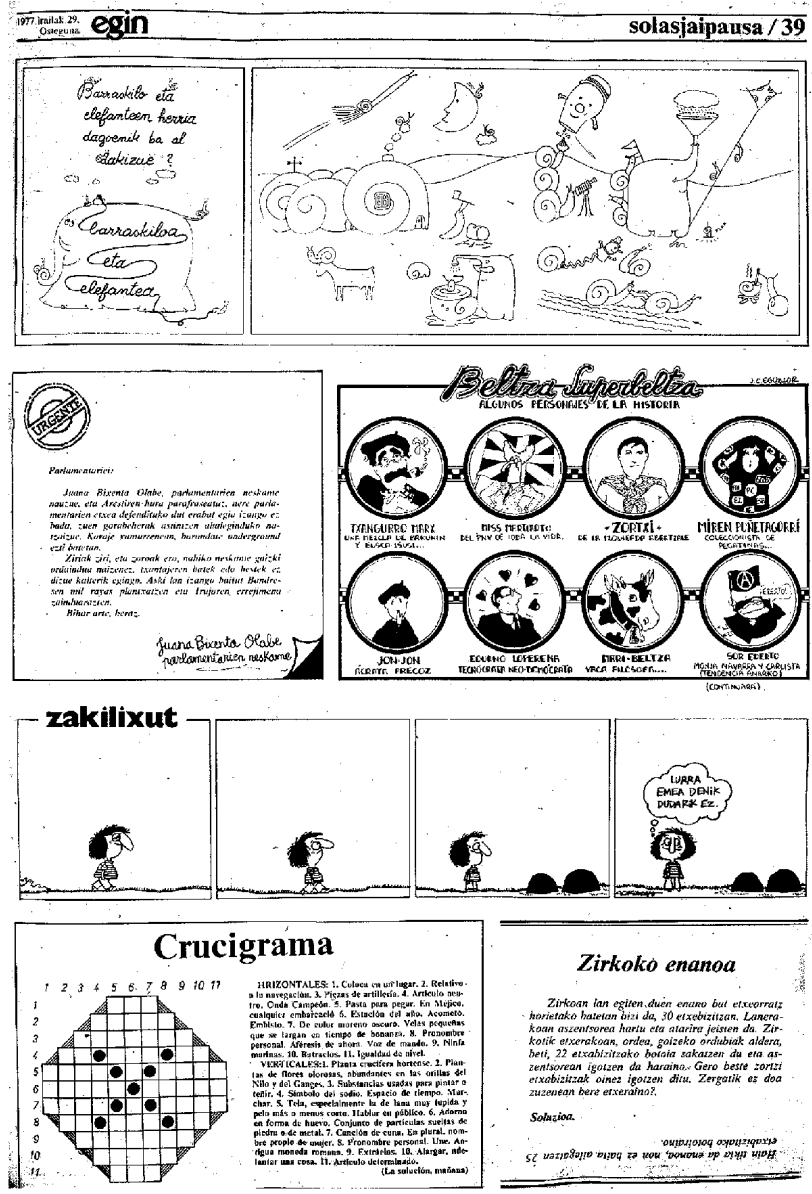

Ilustración 1. Página Solasjaipausa del primer ejemplar de Egin.

[Hemeroteca de la Biblioteca Central del Campus de Leioa (UPV/EHU)]

Solasjaipausa se cierra con una sección de pasatiempos que incluye sopas de letras, crucigramas, acertijos y diversos juegos de ingenio diseñados por Olariaga, tanto en vasco como en castellano. Por tanto, tenemos una página dividida en cuatro secciones, en la que tres cuartos de la misma son dedicadas exclusivamente a la tira cómica.

En lo que refiere al HG, es frecuente encontrarse en las páginas internas del periódico viñetas e ilustraciones de los citados dibujantes, además de otros como Endika y su serie Txingurritxoak ("Las Hormiguitas"). En octubre de 1977, todos los dibujantes tendrán ocasión de publicar en las páginas del periódico cómics didácticos para niños, bajo el nombre colectivo Ipurbeltz [04], donde además 
de los citados, tomarán parte Jesús Lucas, Tomás Hernández Mendizábal, Mikel Plazaola, Alemán Almundarain e Hilario Urkia.

\subsection{El humor gráfico, como disciplina académica}

Se dispone de amplia bibliografía en torno al HG como representación sintomática de una cultura o situación social, habitualmente en períodos conflictivos de la historia que permiten elaborar un discurso en torno a la sociedad retratada. En todos ellos suele ser frecuente la descripción de temas y símbolos reflejados, como es el presente.

No hay que confundir el HG con el cómic. Si bien el primero puede beber de las técnicas expresivas y narrativas del segundo, no todo HG se traslada a la publicación en forma de cómic. En nuestro caso, y como veremos en el apartado metodológico, se muestra como tiras cómicas o series lineales de viñetas, utilizando recursos expresivos de la historieta. Por tanto, y aunque en ocasiones se mezclen, conviene diferenciar la literatura referida al cómic y al HG en particular.

En lo referente al HG, a nivel vasco puede destacarse como principal la obra de JM Tápiz (2002), quien a través de un breve artículo recoge el HG que aparecía en las publicaciones periódicas de mayor tirada en las -entonces denominadas- Vascongadas: Euzkadi, El Liberal, La Gaceta del Norte, Euzkadi Roja y La Lucha de Clases. El autor conviene que las viñetas publicadas en estos diarios eran casi pequeños carteles propagandísticos, convenciendo a sus lectores de determinadas ideas políticas que los sustentaban, tendencia exactamente igual a la seguida en el conjunto de España durante todo el siglo XIX, e incluso en algunos países de América Latina (Ávila, 2002: Presentación, párrafo 4).

De obligada citación es también JM Unsain, quien ha realizado diversas investigaciones sobre el cómic en Euskadi desde la segunda mitad del siglo XIX hasta hoy. Para este estudio, citamos su artículo "El humor gráfico en la prensa de Bilbao y San Sebastián (1865-1936)" (2004), donde identifica a los principales autores y sus obras en la prensa de ambas capitales en el periodo de tiempo especificado. No se trata tanto de una investigación sobre los contenidos, sino recopilación descriptiva de los antecedentes del HG en el territorio, bebiendo de investigaciones previas y propias como su libro “Antecedentes del Cómic en Euskadi” (1990).

A nivel nacional, cabe citar a J Moreiro y su artículo "María de la Hoz: Tono y Mihura en las trincheras" (2007), donde describe la obra artística de Miguel Mihura y Antonio de Lara Tono en el folleto "María de la Hoz", de la revista La Ametralladora durante la Guerra Civil, alentando al bando nacional. Todo ello explicando los chistes gráficos y textos humorísticos contenidos en el mismo.

Derivados de la Transición, como es el caso que nos atañe, encontramos también diversas obras, de las que nos interesan especialmente aquellas que definen la sociedad española y sus conflictos hacia la democracia. Es el caso de Segado Boj, quien partiendo de su tesis doctoral ha publicado diversos artículos, que no citaremos aquí por espacio, pero sí su tesis "La Transición española en el humor gráfico de la prensa diaria (1974-1977)" (2008), en la que combinando ambos métodos cuantitativo y cualitativo, extrae los principales temas de las tiras cómicas y viñetas de cinco periódicos de mayor tirada nacional. No menos interesante resulta la tesis de Meléndez-Malavé "El humor gráfico en el diario El País durante la transición política española (1976-1978)" (2005), donde la autora estudia, durante el período citado, el HG de uno de los periódicos de mayor tirada, al mismo tiempo que analiza las temáticas de las tiras cómicas publicadas y entrevista a sus autores. 
Sin salirnos del ámbito español, hay especialistas como Manuel Barrero, uno de los creadores de la web Tebeosfera (referente en información sobre cómic y HG hispanos -www.tebeosfera.com-), del cual hay que traer un artículo de la revista Mundaiz en el año 2008 sobre unas caricaturas del diario danés Jyllands-Posten, en la que se representaba a Mahoma con símbolos relacionados con la actividad terrorista, lo que desató malestar en el sector islamista radical. Barrero sostiene que es necesario considerar toda imagen humorística en su contexto iconográfico para comprender su significado.

Al hilo del suceso citado, disponemos de una interesante colección de ensayos expuestos en el 2006 por la "International Society for Humor Studies" en Copenhague, y dirigida por P Lewis, donde se analizaron aquéllas caricaturas del profeta; si éstas eran o no propiamente humorísticas y, en caso de serlo, si marcaban o no un nuevo rumbo en la investigación sobre el HG. Siete autores presentaron sus investigaciones, condensados sus comentaros críticos en un simposio de nombre "Transnational Ridicule and Response". Y es que estos ensayos se enmarcan dentro de las investigaciones publicadas en la revista "Humor: International Journal of Humor Research", que desde su aparición en el 88 ha publicado más de 400 artículos sobre humorismo. Referentes al HG, nos interesa destacar a Diners (1995), Forceville (2005) y El-Arousy (2007).

Dando un salto a Norte América desde España, resulta de especial interés el artículo de Francisco Sáez de Adana "Las tiras de prensa norteamericanas de finales del siglo XIX y principios del siglo XX como reflejo de la sociedad" (2014). Durante el período citado, y comenzando desde el famoso Yellow Kid, Sáez hace un recorrido por las páginas de prensa pobladas de cómics, tiras cómicas y caricaturas de los EEUU, identificando por medio de la descripción y el análisis de contenido los estereotipos, clases sociales y tipos sociales reflejados en ellas.

Para cerrar la sección, no podemos dejar de citar a JI Lorente, profesor de la Universidad del País Vasco, quien llevó por primera vez una ponencia sobre el HG de Egin a Francia a través de un congreso celebrado en la Université de Pau et des Pays de l'Adou", de título "El humor y la ironía como armas de combate" (2013). Su comunicación lleva por título "Mirar al sesgo. El humor gráfico de Olariaga y Eguillor en la primera época del diario Egin, donde se analizan las tiras cómicas de ambos autores y se realiza una fotografía de aquella sociedad vasca que ambos reflejan.

\section{Material y estrategias metodológicas \\ 2.1. Algunas definiciones previas}

Aunque ya se ha definido lo que es el HG, utilizando para ello las palabras de Armañanzas y Díaz Noci, debemos concretar antes algunos términos que aparecerán durante el análisis y que no se deben confundir.

La tira cómica, que como hemos dicho antes no es más que una historieta de cómic en sentido lineal, es la manera más usual de HG en publicaciones, pero no la única. En nuestro caso, todas las unidades analizadas del HG de Egin son así. La viñeta, es cada una de las partes que componen estas tiras cómicas, es decir, el encuadre que marca un lapso de tiempo en la historieta (Entrialgo, 2011: 277). A veces, una tira cómica puede ser compuesta de una sola viñeta, como suele ser frecuente en Zabaleta. Así también, existen multitud de formas de estructurar una pieza de HG, como es el caso de Eguillor, quien en vez de utilizar una narrativa lineal, en las que las viñetas siguen una a otra, prefiere una estructura cuadrada, formando un bloque de viñetas. En todo caso, conviene distinguir 
bien todos los términos aquí expuestos, ya que suelen mezclarse. En inglés, suele emplearse "cartoons" o "comic strips".

\subsection{Metodología}

\subsubsection{Muestra}

Se tomaron todas las páginas Solasjaipausa desde el 29 de septiembre al 24 de diciembre del 77. Se descartaron las tiras restantes hasta el fin de año, porque es precisamente a vísperas de nochebuena cuando los dibujantes cierran con sendas tiras cómicas, donde cada uno retrata, a modo de estampa navideña, a sus personajes felicitando a los lectores, lo que supone un corte respecto a la secuencia narrativa hasta la fecha.

El único que no lo hace es Eguillor. Para entender las tiras cómicas de éste, es recomendable leer las publicadas en ejemplares anteriores, cosa que no es necesaria en el caso de Zabaleta y Olariaga. Empero, nos pareció un buen criterio limitar la muestra hasta el día 24, éste último incluido, logrando así un número de cintas con cierta coherencia secuencial y narrativa.

De las páginas, se estudian las tiras cómicas de los tres autores, añadiendo también el texto de JBO, que aunque no se trata específicamente de HG, aporta una opinión que complementa, como veremos, a los anteriores. De aquí en adelante nos referiremos al conjunto muestral como "tiras cómicas" u otros sinónimos por economía del lenguaje, sin perder de vista que englobamos también los textos de JBO.

Se analizaron, por tanto, 73 páginas de Solasjaipausa y 292 tiras cómicas.

\subsubsection{Apartado cualitativo}

El método consiste en la identificación temática de cada pieza gráfica, que por ser en ocasiones muy abstractas (sobre todo en Zabaleta), requieren de particular atención: no es suficiente un golpe de lectura, sino apreciar la interactuación entre los personajes, su entorno, lo que dicen, lo que no dicen, y por supuesto, la actualidad del momento.

El propio diario nos propone unos catalejos concretos, que son la mirada general de la izquierda nacionalista. Si bien no todos los dibujantes presentan necesariamente esta óptica, sí que facilita mucho el punto de partida de los temas que se van a emplear. Del mismo modo, el texto de portada que hemos traído líneas más adelante nos da pistas sobre el tipo de lector, quien también va a leer estas viñetas hasta el punto de comenzar el periódico por detrás. Así pues, se hará también uso de la historiografía, que nos ayudará a situar situaciones sociales determinadas del momento con las imágenes y símbolos que en las tiras cómicas se reflejan.

No es pretensión nuestra hacer un listado de todos los elementos narrativos de cada tira, pero sí, impregnados de la actualidad expresada por el periódico, plantear una lista de temáticas que estructuran las mismas y que en muchos casos serán una constante en los autores.

También hay que tener en cuenta que en cada pieza puede hallarse más de un tema, en tanto que por tratarse de aspectos diversos de una sociedad plural, a menudo una temática puede llevar a otra y complementarla. La necesidad de síntesis nos ha llevado a identificar por cada pieza, un solo tema, 
eligiéndose en cada caso el más sobresaliente. El listado de temas obtenidos, se enuncian en una frase breve, casi sintagmática, con intención de facilitar la legibilidad y esquivar la ambigüedad.

\subsubsection{Apartado cuantitativo}

Nos interesa saber qué temas son los más recurrentes por los autores, y en cada caso, cuáles son los más utilizados por cada uno. Pretendemos un apartado cuantitativo, donde se pueda recoger en cuántas tiras cómicas aparece cada tema identificado, así como con qué frecuencia cada tema aparece en la obra de cada autor.

Preparamos una tabla en Excel, donde se sintetizan los datos de la siguiente manera:

\begin{tabular}{|l|l|l|l|l|}
\hline Página Solasjaipausa y fecha & \multicolumn{4}{l|}{} \\
\hline Serie & $\begin{array}{l}\text { Barraskiloa eta } \\
\text { Elefantea }\end{array}$ & Beltza-Superbeltza & $\begin{array}{l}\text { Juana Bixenta } \\
\text { Olabe }\end{array}$ & Zakilixut \\
\hline & $($ BeE) & (BSB) & (JBO) & $(\mathrm{Z})$ \\
\hline Tema 1 & 1 & 0 & 0 & 0 \\
\hline Tema 2 & 0 & 1 & 1 & 0 \\
\hline Tema 3 & 0 & 0 & 0 & 1 \\
\hline$\ldots$ & $\ldots$ & $\ldots$ & $\ldots$ & $\ldots$ \\
\hline
\end{tabular}

Tabla 1. Tabla de análisis. Elaboración: Propia

En cada casilla se añade un 0 o un 1 dependiendo de si el tema en cuestión se muestra o no en cada tira cómica estudiada. Una vez rellenada toda la tabla, pueden efectuarse las sumas de cada tema, obteniendo las frecuencias que se requieran, tanto en lo que refiere a los temas en concreto, como a la presencia en cada autor.

\section{Resultados}

Se identificaron un total de 49 temas, algunos muy similares entre sí. Por tratarse de una lista excesivamente larga, se decidió sintetizar lo recolectado en 14 bloques, un tanto menos específicos que los temas en particular, pero que marcan una serie de ejes de las distintas temáticas empleadas por los autores:

a) Bloque 1 (B.01). - Reivindicación de la democracia: Todas aquellas tiras cómicas en las que se refleja la necesidad de una solución democrática y participación de los pueblos en la misma 56 unidades $(\mathrm{U})$.

b) B.02. - Desplazamiento del nacionalismo tradicional: Si el nacionalismo en su vertiente clásica y fuerista había sido católico y partidario de la reintegración de la reintegración foral plena ("Lagi zaŕak"), las nuevas tendencias abertzales apuestan por la vía de la izquierda, el marxismo y el anticlericalismo, dejando de lado las tesis del nacionalismo primigenio.

$47 \mathrm{U}$

c) B.03. - Progresismo VS conservadurismo: Antítesis entre las nuevas tendencias sociales hacia la democracia y entre las posturas conservadoras, sobre todo en el ámbito religioso $42 \mathrm{U}$ 
d) B.04. - La cuestión Navarra: Se debate a nivel político el estado de las autonomías, que en el caso vasco, se entremezcla con las luchas obreras y el derecho de autodeterminación. Sobre este eje, se integran posturas que consideran a una Euskalerría que incluye a Navarra, y quienes entienden que dicha provincia debe constituir un sujeto propio dentro de la nación española, a través de su tradición foral [5] Esta última postura es fuertemente identificada en Egin como postura de la extrema derecha y el conservadurismo

$35 \mathrm{U}$

e) B.05. - Ambiente político de Euskadi: La inestabilidad política del País Vasco es reflejada en las tiras cómicas como la imposibilidad ocasional de alcanzar acuerdos entre iguales

$25 \mathrm{U}$

f) B.06. - Otros: Temas que no pueden ser clasificados en los demás bloques. Entre ellos hay experimentos que algunas veces los dibujantes hacen con las formas, como es frecuente en Zabaleta.

$19 \mathrm{U}$

g) B.07.- Feminismo: Temáticas en torno a la presencia pública de la mujer y su liberación social. $14 \mathrm{U}$

h) B.08. - El poder coercitivo: Reflejo del poder central autoritario aún latente e insuperado. $13 \mathrm{U}$

i) B.09. - Derecho de autodeterminación: Reivindicaciones del derecho de Euskalerría a ser independiente.

$12 \mathrm{U}$

j) B.10. - Dos modelos de izquierda: Contradicción entre la izquierda nacional y la nacionalista, que confluyen en todo menos en la referencia nacional.

$10 \mathrm{U}$

k) B.11. - Reivindicación del euskera: Apoyo a las primeras "ikastolas" (escuelas vascas) y a la enseñanza libre y oficial del idioma.

$5 \mathrm{U}$

1) B.12. - Actualidad: Temas que obedecen estrictamente a sucesos momentáneos. $5 \mathrm{U}$

m) B.13. - Relaciones Iglesia-Estado: Debate de la presencia que debe tener la confesionalidad en el estado incipiente, tema de discusión en las Cortes Españolas que se salda con duras críticas sobre todo por parte de la izquierda laicista

$5 \mathrm{U}$

n) B.14. - Desafío de la modernidad: Recelo de la modernidad como vía al capitalismo y a la destrucción de tradiciones vascongadas que, si bien no son el núcleo ideológico de la IA, sí que son consideradas.

$1 \mathrm{U}$ 
En lo que refiere a las series dibujadas por los autores, puede apreciarse la siguiente frecuencia de las temáticas utilizadas:

\section{Número total de piezas gráficas por series}

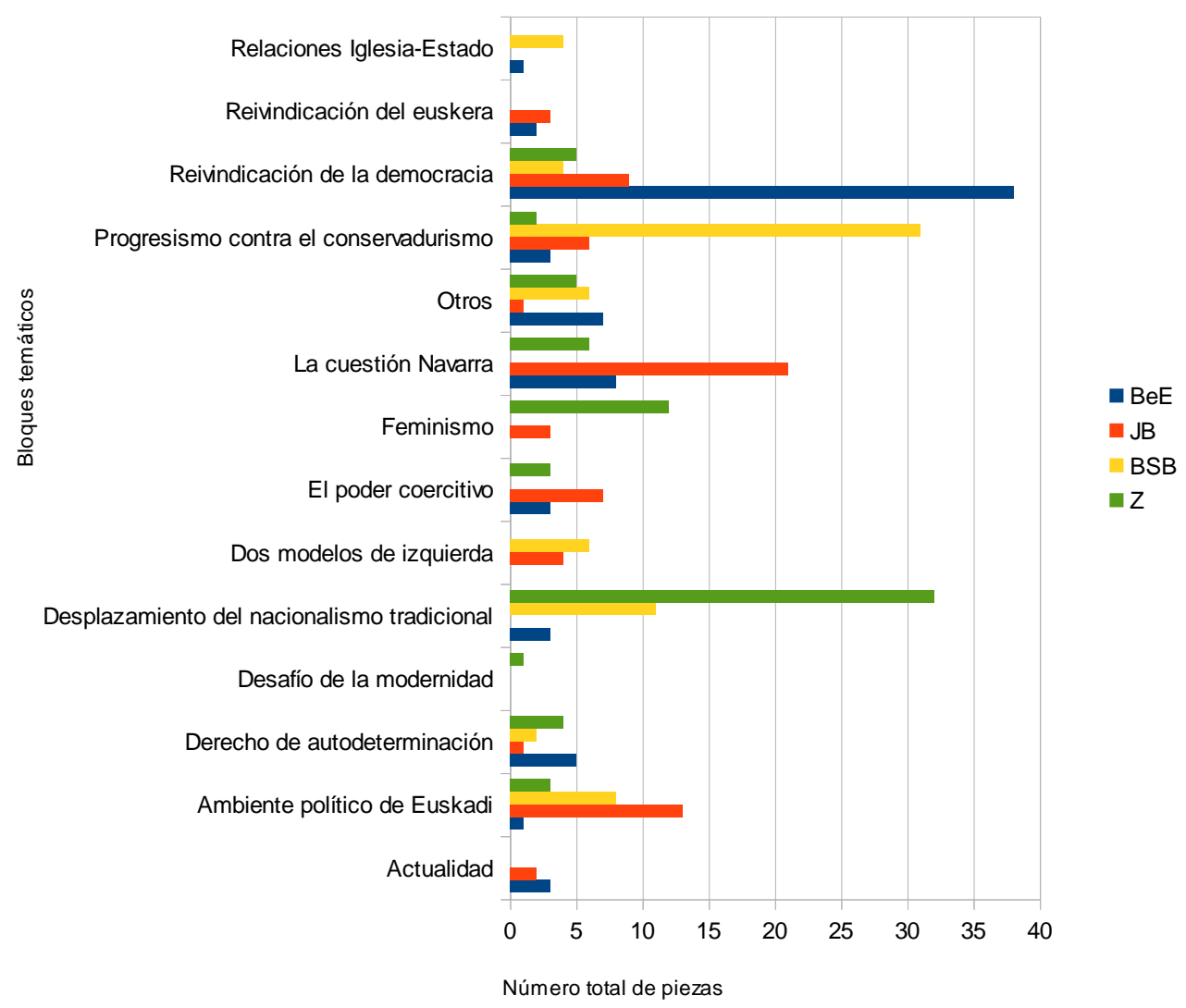

Gráfico 1. Número total de piezas gráficas por series. Elaboración: propia.

Como puede apreciarse a simple golpe de vista, es Zabaleta (BeE) quien más frecuentemente recurre a los temas relacionados con la reivindicación democrática, que ya hemos visto, son también los más frecuentes.

El desplazamiento del nacionalismo tradicional queda casi patrimonializado por el Zakilixut (Z) de Olariaga, mientras que la antítesis progresismo-conservadurismo es más propio de la "ciudad negra" de Eguillor (BSB).

La cuestión Navarra y el caldeado ambiente político vasco son frecuentemente tratadas por JBO, es muy dura con la UCD de Navarra, contraria a la integración de la provincia en el proyecto "euzkadiano".

\section{Discusión y conclusiones}

Resulta curioso cómo los temas que más se compromete a tratar Egin desde sus comienzos no constituyan el grueso de la temática de los dibujantes, en tanto que se vuelcan prioritariamente a favor de la democracia, que no atañe sólo al pueblo vasco, sino al español en su conjunto. 
La reivindicación del euskera como lengua nacional queda tal vez 'reducida' al lenguaje con el que al menos Olariaga y Zabaleta expresan sus ideas, en tanto que la reivindicación de la lengua como tal constituye sólo un $1^{\prime} 70 \%$ de los temas tratados y cinco piezas estudiadas.

Curioso es también el modelo de nacionalismo que los dibujantes parecen querer impulsar: casi un $20 \%$ de la temática gira en torno a enfrentar las tesis nacionalistas clásicas contra las nuevas tendencias de la IA, más ligada a la lucha obrera y al marxismo. El fuerismo clásico, apartado a la órbita de la derecha conservadora, es aplastado por casi un $15 \%$ de las unidades estudiadas. La cuestión territorial parece volcarse sobre Navarra, que parece preocupar notoriamente a la muy observadora JBO. La actualidad más ceñida no llega ni a un $2 \%$ de la frecuencia.

Puede concluirse que la temática de las tiras cómicas estudiadas está en total sintonía con la filosofía del primer consejo de dirección del diario Egin, que pretende llegar a un amplio sector de la izquierda vasca, sin importar tanto si es o no nacionalista, aunque sí se hace hincapié en la cuestión territorial, donde indiscutiblemente, ese "pueblo obrero trabajador vasco" está también en una Navarra que la derecha desea mantener "sui géneris". A los "nuevos nacionalistas" los alejan de las tendencias de hasta el momento, integrándolos en un nuevo proyecto de país del que Egin es partícipe.

\section{Notas}

[1] En aquel momento existían, bajo la tutela de la Prensa del Movimiento, los diarios El Correo Español - El Pueblo Vasco, La Gaceta del Norte, La Voz de España, El Diario Vasco, Unidad, "Hierro" y Norte Exprés. Los dos primeros contaban con su sede principal en Bilbao, teniendo El Correo ediciones locales en Álava, la zona guipuzcoana Éibar-Mondragón, La Rioja y Miranda de Ebro. La Gaceta se extendía por Vizcaya, Álava, Navarra, La Rioja y Cantabria. Ninguno corresponde con el ámbito regional vasco. Los restantes periódicos podrían denominarse “provinciales": La Voz, Diario Vasco y Unidad se difundían en Guipúzcoa, y Norte Exprés en Álava, con sede en Vitoria. Hierro era un diario local bilbaíno.

Complementaban los lectores su nivel informativo con las denominadas Hoja del Lunes, publicaciones locales que salían a la calle el primer día de la semana, día de descanso de la prensa oficial. A través de las mismas, los periodistas podían esquivar la Prensa del Movimiento y publicar noticias con un tono menos cauteloso. Así tenemos sendas Hoja del Lunes en Bibao y San Sebastián, con un promedio de difusión de 93000 y 72000 ejemplares respectivamente (Coca \& Fernández, 1993: 15).

[2] Históricamente, y en grafía castellana, se ha venido denominando "Euskalerría" al conjunto de territorios de habla vasca, entendiendo como tales las actuales comunidades autónomas del País Vasco y de Navarra, en España, y las comarcas del "Labourd" ("Lapurdi", en euskera), la "BasseNavarre" o Baja Navarra ("Nafarroa Beherea") y "Soule" ("Zuberoa") en el departamento francés de los Pirineos Atlánticos. Actualmente es de común uso el término "Euskal Herria" ("pueblo vasco" o "país del euskera").

[3] Eran los tiempos en los que se debatía el estatuto de autonomía: mientras los diputados regionales del PNV, el espectro de la IA y la izquierda en general aceptaban un régimen estatutario conjunto para las Vascongadas y Navarra, no así el espectro de la derecha, sobre todo representada por UCD y Alianza Popular, quienes pretendían un estatuto separado para ésta última en relación a su vigente 
régimen foral. ETA no dudó en perpretrar diversos atentados al respecto, uno de los cuales segó la vida del teniente-coronel Ímaz, ligado a la Policía Armada de Navarra. El 28 de diciembre del 77 tenía lugar el atentado, y al día siguiente se publicó el controvertido texto que terminó también con el cargo de Ferrer (Egin, 29/12/1977, p. 5).

[4] El adjetivo "ipurbeltz" puede traducirse por "culo negro", y evoca un antiguo proverbio vasco ("atxotitza") que dice "xoxuak beleari ipurbeltz" (el mirlo le dijo al cuervo "culo negro"), muy próximo a la expresión castellana "le dijo la sartén al cazo". Sin duda un nombre que marca al carácter señalador de estos humoristas, más cerca de la burla y la sátira que del humorismo limpio que se venía practicando en la prensa española desde el franquismo. Buen ejemplo de ello es el conocido "Don Celes", de El Correo, o la primera etapa de La Codorniz, bajo la dirección de Mihura.

[5] La literatura sobre el derecho foral es extensa, y delicada en terreno político. Mientras el nacionalismo clásico, encarnado por el PNV, ha considerado los derechos forales o históricos símbolo de una antigua independencia destruida por el liberalismo, el tradicionalismo y el carlismo los han tomado como derechos propios de las provincias y villas españolas, otorgados y ratificados por el rey, que recogen los usos y costumbres de los territorios y los integra en la nación. Hasta hoy, ambas tesis han sido irreconciliables en el País Vasco, siendo la una propia del PNV, y la otra de las distintas ramas de la derecha española, con matices intermedios. Ambas son, con diferencias, más o menos autonomistas.

\section{Referencias bibliográficas}

C Abreu (2001): "Periodismo iconográfico (VII). Hacia una definición de caricatura". Revista Latina de Comunicación Social, 40. Recuperado el 28 de octubre de 2015 de:

http://www.ull.es/publicaciones/latina/2001/latina40abr/102cabreuVII.htm

E Armañanzas \& J Díaz Noci (1996): Periodismo y argumentación. Géneros de opinión. La opinión iconográfica, 111-117. Bilbao: Universidad del País Vasco.

X Ávila (2002): La caricatura, en el periodismo del nuevo siglo. Revista Latina de Comunicación Social, 46. Recuperado el 28 de octubre de 2015 de:

http://www.ull.es/publicaciones/latina/2002/latina46enero/4608ximena.htm

M Barrero (2008): "La controversia de las viñetas de Mahoma. Géneros, alcance y propaganda en la sátira gráfica". Mundaiz, 75, 113-158. Recuperado el 24 de septiembre de 2015 de:

http://www.tebeosfera.com/documentos/textos/la_controversia_de_las_vinetas_de_mahoma_generos _al-cance_y_propaganda_en_la_satira_grafica.html

V Bécquer \& GA Bécquer (1991): SEM. Los Borbones en pelota. Madrid: El Museo Universal.

C Coca \& F. Martínez (coord.) (1993): Los medios de comunicación en el País Vasco. Leioa:

Universidad del País Vasco.

J Díaz Noci (2012): "Historia del periodismo vasco (1600-2010)". Mediatika. Cuadernos de medios de comunicación, 13, 1-261. Recuperado el 25 de noviembre de 2015 de:

http://dialnet.unirioja.es/servlet/articulo?codigo $=4366001 \&$ orden $=409179 \&$ info $=$ link 
G Diners (1995): "Towards a Critical Sociological Analysis of Cartoons". Humor - International Journal of Humor Research, 3(VIII), 237-255. DOI: 10.1515/humr.1995.8.3.237

NA El-Arousy (2007): "Towards a Functional Approach to the Translation of Egyptian Cartoons". Humor - International Journal of Humor Research, 3(XX), 297-321. DOI:

10.1515/HUMOR.2007.015

M Entriaglo (2011): "Diario de un taller de humor gráfico e historieta costumbrista". ARBOR Ciencia, Pensamiento y Cultura, 170, 275-283. DOI: 10.3989/arbor.2011.2extran2124

C Fernández de la Vega (2002): O segredo do humor. Segunda Edición. Colección Biblioteca Galega. A Coruña: La Voz de Galicia

C Forceville (2005): “Addressing an Audience: Time, Place, and Genre in Peter Van Straaten's Calendar Cartoons”. Humor - International Journal of Humor Research. 3(XVIII), 247-278, DOI: 10.1515/humr.2005.18.3.247

GFA (Gipuzkoako Foru Aldundia) (1998): Antton Olariaga. Euskara Gipuzkoan. Recuperado el 3 de enero de 2012 de: http://www.gipuzkoaeuskara.eus/jarduerak/abbadia/olariaga

L Gil Fernández (1996): Aristófanes. Madrid: Gredos

JA Llera (2003): El humor verbal y visual de “La Codorniz”. Madrid: CSIC

S López (2005). Sobre o humor de Cervantes no Quixote. Colección Cuadernos de Ramón Piñeiro (IX). Santiago de Compostela: Xunta de Galicia

E Lorente (2015): "Mirar al sesgo. El humor gráfico de Olariaga y Eguillor en la primera época del diario Egin”. In A Fernández Ferrer (dir.) (2015): El humor y la ironía como armas de combate. Libro de actas, 530-552. Sevilla: Renacimiento.

JC Mainer \& S. Juliá (2000): El aprendizaje de la libertad. Madrid: Alianza

N Meléndez Malavé (2005): El humor gráfico en el diario "El País” durante la transición política española (1976-1978). Tesis Doctoral. Málaga: Universidad de Málaga

J Moreiro (2007): "María de la Hoz: Tono y Mihura en las trincheras". Anales de Literatura Española, 19, 161-172. Recuperado el 3 de enero de 2012 de:

http://rua.ua.es/dspace/bitstream/10045/7219/1/ALE_19_09.pdf

OJD (1981): Egin. No 187, p. 21

R Reig (2008): "El poder del humor y el humor bajo el poder". Revista Latina de Comunicación Social. Recuperado el 28 de octubre de 2015 de:

http://www.revistalatinacs.org/08/humor/Ramon_Reig.html

JP Richter (1812): Las teorías estéticas (Traducción: Julián de Vargas). Tomo XV. Madrid: Sociedad General Española de Librería 
F Sáez de Adana (2014): "Las tiras cómicas de prensa de finales del siglo XIX y principios del siglo XX como reflejo de la sociedad". Revista internacional de Historia de la Comunicación, 3(I) 20-47. Recuperado el 24 de septiembre de 2015 de: http://dialnet.unirioja.es/descarga/articulo/4918002.pdf

F Segado Boj (2008): La Transición española en el humor gráfico de la prensa diaria (1974-1977). Tesis doctoral. Madrid: Universidad Complutense.

JM Tápiz (2002): "El humor político en la prensa vasca durante la II República”. Sancho el sabio: Revista de cultura e investigación vasca, 17, 55-66. Recuperado el 24 de novirmbre de 2015 de: http://dialnet.unirioja.es/descarga/articulo/298075.pdf

I Tubau (1973): De Tono a Périch. El chiste gráfico en la prensa española de la posguerra (1939 1969). Madrid: Fundación Juan March Guadarrama.

JM Unsain (1990): Antecedentes del cómic en Euskadi (1894-1939). San Sebastián: Ttarttalo S.A.

JM Unsain (2004): "El humor gráfico en la prensa de Bilbao y San Sebastián (1865-1936)". Ondare, 23, 599-614. Recuperado el 3 de enero de 2015 de: https://www.euskoikaskuntza.org/es/publicaciones/colecciones/cuadernos/articulo.php?o=9435

JM Unsain \& CC Borra (s.f.) “Aproximación a la historia del cómic en Euskadi (1936 - 2004)”. Etor-Ostoa: La enciclopedia emblemática. Artes Aplicadas (II). Recuperado el 10 de julio de 2007 de: http://www.etorkultura.com/capitulos/T-22.pdf

\section{Cómo citar este artículo / Referencia normalizada}

A Castañeda Zumeta, P Pineda-Martínez (2016): "La reivindicación democrática a través de las viñetas. Las temáticas del humor gráfico en el periódico vasco Egin (1977)”. Revista Latina de Comunicación Social, 71, pp. 232 a 248.

http://www.revistalatinacs.org/071/paper/1093/13es.html

DOI: $\underline{\text { 10.4185/RLCS-2016-1093 }}$

- En el interior de un texto:

... A Castañeda Zumeta, P Pineda-Martínez (2016: 232-248)...

Artículo recibido el 18 de enero de 2016. Aceptado el 27 de febrero.

Publicado el 21 de marzo de 2016. 